\title{
Anoxic Tonic Seizures Due to Asthma; A Serious Complication in Adults
}

\author{
D.L. KEENE, C.A. MELMED, F. ANDERMANN and D.W. BAXTER
}

SUMMARY: Anoxic tonic seizures are reported as a complication of severe asthma in two adults. The nature of these attacks can be misinterpreted. They do not indicate the presence of a recurrent seizure problem unconnected to the episodes of anoxia caused by asthma. These attacks do not respond to antiepileptic medication. but cease if the asthma can be controlled. One of our patients developed an anoxic encephalopathy and the other died. The need for prompt diagnosis and appropriate treatment is emphasized.

RESUME: Les crises toniques suite d̀ l'anoxie sont signalées comme étant une complication sévère de l'asthme. La nature de ces crises peut être mal interprétée. Elles ne sont pas épileptiques dans le sens qu'elles n'indiquent pas la présence d'un problème récidivant d'épilepsie non associé ou associé seulement en partic aux épisodes d'anoxie causés par l'asthme. Ces attaques ne cèdent pas à une médication antiépileptique mais cessent si l'asthme peut être contrôlé. Un de nos patients a développé une encéphalopathie anoxique et l'autre est décédé. Un diagnostic précoce ainsi qu'un traitement approprié s'imposent.

From the Department of Neurology and Neurosurgery, McGill University

Presented in part at the Fourteenth Canadian Congress of Neurological Sciences. Halifax, Nova Scotia, June 1979

Reprint requests to Frederick Andermann, M.D., Montreal Neurological Hospital and Institute, 380I University St., Montreal, Quebec, Canada H3A 2B4

\section{INTRODUCTION}

Tonic attacks or anoxic convulsions are common in association with breath holding or vasovagal attacks in children and have also been recognized as a complication of childhood asthma. These attacks have not been described in asthmatic adults. We report two patients who initially presented a problem in diagnosis; one eventually developed an anoxic encephalopathy and the other died during an attack.

\section{CASE REPORTS}

\section{Case 1:}

R.A. - A 29 year old female school teacher developed wheezing at 12 months of age. At age 4, breath holding spells were noted and at age 7 she experienced her first attack of loss of consciousness clearly associated with asthma.

At age 23 , her asthmatic attacks increased in severity and frequency. More severe attacks triggered loss of consciousness. She was diagnosed as an epileptic. Neurologic examination, skull $x$-ray, and a routine EEG were normal. Phenytoin $300 \mathrm{mg}$. q.d. was prescribed but because of skin rash and subjective intolerance was discontinued, and mephobarbital $300 \mathrm{mg}$. q.d. was started.

Over the next 4 years she continued to experience stereotyped nocturnal attacks of wakening with shortness of breath, coughing, and a sensation of being about to lose bladder control, followed by loss of consciousness. Tonic stiffening of the body, often accompanied by tonque biting and urinary incontinence was observed. Afterwards she was somnolent. During this period her anticonvulsants were continued, but were ineffective.

At age 27 , she experienced an increased frequency of acute asthmatic attacks. Delta cortisone, oxtriphylline and a salbutamol inhaler were continued. Another routine EEG was normal. Carbamazepine $600 \mathrm{mg}$. q.d. was added to her regime. Milder diurnal attacks of asthma, unassociated with loss of consciousness, continued. Over the next 6 months, associated with a decrease in delta cortisone dosage, more frequent attacks of nocturnal asthma and tonic attacks occurred. Primidone $750 \mathrm{mg}$. q.d. was added to her anticonvulsant regime. She showed evidence of drug intoxication with somnolence, nystagmus and ataxia. Her attacks continued to increase in frequency. This period culminated in a severe attack of asthma followed by one of her habitual tonic seizures, as well as respiratory arrest and subsequent anoxic encephalopathy.

During recovery she had severe memory impairment, as well as limb and gait ataxia. EEGs in the early postanoxic period showed diffuse slowing, gradually returning to normal. Clinically she continued to improve but still showed mild memory and behavioral disturbances. CT scan was normal.

With improved control of her asthma there have been no subsequent severe attacks or tonic seizures. She still receives $150 \mathrm{mg}$. of phenytoin daily (serum level llug./ml.), which she is reluctant to discontinue.

\section{Case 2:}

D.L. - A 36 year old male had a 20 year history of severe asthma requiring treatment with high doses of steroids, oxtriphylline, disodium chromoglycate and salbutamol.

At age 23 he was admitted to another hospital after being struck over the head in a fight. He was unconscious for three days. No details of that admission are available, but no 
fracture was reported and no surgery was preformed. He was discharged with no known residual neurological changes.

At age 36 he had his first nocturnal attack. He awoke from sleep with wheezing followed by unresponsiveness and tonic posturing. Two similar attacks subsequently occurred before his admission to hospital three months later. During the last episode, jerking of the right side of the body was described. After this he had a mild right hemiparesis which cleared over 3 days. Skull $x$-rays, CT scan and two EEGs, one obtained following sleep deprivation, were normal. A diagnosis of epilepsy was made. Anticonvulsant medications, which had been started on admission to hospital, were discontinued after 48 hours in preparation for further electrographic investigation. Delta cortisone was maintained throughout his hospitalization at $5 \mathrm{mg}$. q.i.d. In addition, oxtriphylline 200 mg. q.i.d., disodium chromoglycate caps 1 q.i.d., and salbutamol 2 puffs q.i.d., were also administered.

On the sixth day of his hospitalization, he became increasingly agitated and began to hallucinate. Sixteen hours later increasing respiratory distress suddenly developed and did not respond to subcutaneous adrenalin and salbutamol. Progressive respiratory difficulty led to unresponsiveness and he had a prolonged tonic seizure. Bradycardia ensued followed by complete heart block. Cardiopulmonary resuscitation was unsuccessful.

Post-mortem examination of the lungs showed mucosal blocking,eosinophilic infiltrates with submucosal edema compatible with status asthmaticus. The brain was unremarkable except for a small superficial glial scar of the left anterior temporal cortex compatible with the effect of remote craniocerebral trauma.

\section{DISCUSSION}

In these two patients, a clear relationship existed between the episodes of acute severe asthma and the occurrence of tonic seizures. Control of the asthma led to cessation of the seizures in the first patient, whereas maximal doses of antiepileptic medication failed to alter their frequency or severity. Less severe asthmatic attacks were not accompanied by loss of consciousness or seizures. In the second patient, the attacks were also preceded and ushered in by an asthmatic event. These observations suggest that the asthma triggered the attacks rather than that the respiratory distress represented an aura of partial seizures as was initially suspected in both patients. Shortness of breath is an unusual symptom of an epileptic aura, though tickling or other sensations in the throat are relatively common.

The role of theophyllin-like medications in lowering seizure threshold is well known, but probably was not significant in our patients.

The etiology of the seizures appeared to be acute nonischaemic hypoxia associated with severe asthma. In the first patient all witnessed convulsions were tonic and of short duration, quite similar to anoxic convulsions described by Gastaut et al. (1957) following syncope. No other epileptic manifestations were noted and there was no electrographic epileptic discharge though the investigation was not exhaustive. No attacks were recorded. Similarly, the second patient also had tonic attacks induced by acute exacerbation of his asthma. In one seizure, however, some lateralizer jerking of the right extremities was described and he had a transient hemiparesis following this event. This may have represented an epileptic seizure, but the description by his wife is unclear, and the subsequent hemiparesis may have been due to anoxia.

Acute nonischemic anoxia as a cause of seizures in asthmatic children was suggested by Nellhaus et al. (1975), who reviewed a series of 342 patients. Of these $9.4 \%$ had seizures which were thought to be related to their cyanotic asthmatic attacks. The authors did not distinguish between tonic or anoxic convulsions and epileptic attacks. Some of these children later developed recurrent epileptic seizures presumably due to the effect of previous repeated anoxia, but in the majority the attacks ceased when the asthma was controlled.

Gastaut and Fischer-Williams (1957) provided a detailed analysis of acute ischemic hypoxia in non-epileptic humans. After seven seconds of ocular compression, bradycardia or asystole and hypotension ensued. After a further seven seconds of asystole, asynchronous theta and delta activity in the EEG associated with mild impairment of consciousness was observed. If asystole was longer than 14 seconds, myoclonic jerks occurred followed by decerebrate posturing. This decerebrate posturing consisted of intense extensor tonic spasms with opisthotonus. The EEG pattern during the tonic extension showed low voltage fast activity similar to what has been described in decerebrate attacks by Celesia and Andermann (1964). With return of heartbeat, consciousness returned.

Hypoxemia with intact perfusion (non-ischemic hypoxia), an abnormality similar to severe asthma may be produced by inhalation of high nitrogen - low oxygen mixtures. In several hundred human patients without epilepsy, Gastaut et al.'s (1961) findings were similar to those following ischemic hypoxia. In epileptics this technique induced activation of both focal and generalized epileptogenic electrographic discharges, however there is no report of induction of clinical attacks.

Thus, the distinction between anoxic convulsions and epileptic seizures may be difficult and only intensive recording and monitoring of attacks may clarify the issue. It was obviously impossible to do this in these two patients. The pathophysiological nature of the attacks suffered by the patients reported here remains undetermined. They may be due to generalized neuronal discharge precipitated by anoxia or represent decerebrate attacks due to inactivation by anoxia of forebrain mechanisms. Thus, anoxic and epileptic convulsions may share certain neurophysiological mechanisms. In the second patient anoxia may have activated epileptic discharge related to the patient's temporal lesion.

Further attempts to classify the attacks of our patients would remain speculative, clearly however an asthmatic event ushered them in in each case.

Both patients reported suffered anoxic damage following respiratory arrest and tonic seizures leading in the 
first to diffuse encephalopathy, and in the second to transient hemiparesis and later to cardiac arrest.

The occurrence of anoxic seizures in adults with severe asthma should be recognized. These seizures present a problem in differential diagnosis. Efforts should be directed toward the control of the asthma since the seizures are a warning of impending serious consequences and since anti-epileptic medication alone does not appear to be effective in the treatment of these attacks.

\section{REFERENCES}

CELESIA G, ANDERMANN F. (1964). Some observations on the elect rographic correlates of the decerebrate attack. Electroencephalog Clin Neurphysiol 16, 295-300.

GASTAUT H, FISCHER-WILLIAMS M.
(1957). Electroencephalographic study of syncope. Lancet I, 1018-1025.

GASTAUT H. GASTAUT F, FERNAN DEZ-GUARDIOLA A, et al. (1961) Hypoxic activation of EEG by nitrogen inhalation. In Gastaut $H$ and Meyer JS (Eds): Cerebral Anoxia and Electroencephalogram. C. Thomas, Springfield, pp 343-355.

NELLHAUS G, NEUMAN I, ELLIS E, et al. (1975). Asthma and Seizures in Children. Pediatr Clin North Am 22(1), 89-100. 